\title{
La administración de la investigación en la Universidad Estatal a Distancia de Costa Rica: revisión histórica desde 1977 hasta 2006
}

\author{
Maynor Barrientos Amador \\ Investigador de la Vicerrectoría de Investigación, UNED. Coordinador del Grupo de Investigación para la Promoción del \\ Trabajo en Red (ProRed). Correo electrónico: mbarrientos@uned.ac.cr
}

Recibido: Febrero 2014 • Aceptado: Mayo 2014

\section{RESUMEN}

Este artículo llena un vacío institucional al poner en perspectiva histórica la evolución de la administración de la investigación en la UNED. Por medio de una exhaustiva revisión documental y una comparación analítica, se establecen cinco etapas de evolución bien diferenciadas marcadas por el desarrollo de congresos universitarios, la promulgación de políticas y el desarrollo y colapso de diferentes unidades académicas y administrativas relacionadas con la investigación en la UNED. El estudio se realizó durante el 2006 y sirvió como antecedente a la propuesta de creación de la Vicerrectoría de Investigación de la UNED, que comenzó a funcionar en 2007. Se evidencia que la historia de la investigación en la UNED ha acompañado la historia misma de la Universidad y ha estado vinculada de forma directa o indirecta a hitos universitarios de trascendencia, a la generación de documentos y discursos diversos, y a la transformación de instancias relacionadas con la investigación en la UNED a través de los años. Se espera que este artículo sirva de referencia a futuros estudios relacionados con la temática de la administración de la investigación en la UNED.

Palabras clave: investigación, UNED, sistema de investigación, historia, educación a distancia.

\begin{abstract}
This essay gives a historical perspective of the evolution of research management at UNED. It established five well-differentiated historical stages and an analytical comparison through an exhausting bibliographical review related to university congresses, policy development, and the creation and collapse of various administrative and academic structures related to research activities at UNED. This study was developed in 2006 and became the documental antecedents for the project that proposed the creation of the Research Department at UNED, a department that started functioning in 2007. It shows how UNED's research history developed along with the university general history, and how it has been linked, directly or indirectly, to transcendental university milestones, the generation of documents and discourses, and to the transformation of all the entities related to research at UNED through the years. This article is expected to work as a reference to future studies related to research management at UNED.
\end{abstract}

Key words: research, UNED, research system, history, distance education. 


\section{Primera etapa: los primeros años}

A pesar de que su génesis es casi contemporánea a la de la Universidad Nacional (UNA) y a la del Instituto Tecnológico de Costa Rica (ITCR), la UNED mantiene un rezago en materia de investigación general, científica y de innovación tecnológica. Esto a pesar de que desde su creación, el 12 de marzo de 1977, se incluye el quehacer de la investigación entre sus objetivos fundamentales.

La Ley de Creación de la UNED (UNED, 1976), en su artículo 2, incisos d) e i), explícitamente dice: "d) Contribuir a la investigación científica para el progreso cultural, económico y social del país [...] i) Fomentar el espíritu científico, artístico, cultural y cívico del pueblo costarricense". Y entre sus funciones fundamentales, en relación con la investigación, es clara en decir que la UNED debe "b) Desarrollar programas de investigación en áreas fundamentales para el desarrollo del país".

Después de su creación en 1977, los tres primeros años fueron regidos, como lo establece la Ley de Creación, por una junta universitaria, la cual se dedicó a organizar todos los aspectos básicos de estructura y organización de la nueva universidad, siendo punto prioritario el diseño de programas de carrera y cursos para ofrecerlos a la comunidad costarricense. Como parte del primer organigrama aprobado entre 1977 y 1979, se propuso el primer Centro de Investigaciones adscrito en teoría a la Rectoría, mientras que en lo concerniente a la investigación institucional necesaria para los procesos importantes en los inicios de la Universidad, desde 1977, se contaba con un número considerable de unidades especializadas adscritas a la Vicerrectoría de Planificación (Junta Universitaria, 1979). Esta Vicerrectoría estaba conformada, en un primer nivel, por el Centro de Documentación (CIDI) y el Centro de Investigación y Estadística (CIEST) $\mathrm{y}$, en un segundo nivel, por oficinas relacionadas con el planeamiento administrativo y con el planeamiento académico. Es dentro de esta segunda categoría que se contaba con las primeras oficinas relacionadas estrictamente con lo que en la universidad históricamente se ha llamado investigación institucional; estas eran dos oficinas: la Oficina de Programación Curricular y la Oficina de Investigación Institucional.

El 27 de setiembre de 1977 se aprueban las primeras políticas de investigación institucional de la UNED, en la sesión $n .^{\circ} 35$, artículo VI, de la Junta Universitaria. Es en este documento histórico donde la investigación institucional se establece como prioritaria en la UNED, en relación con otros tipos (general, científica, educativa). Se dice literalmente: "Los procesos de investigación en la UNED estarán dirigidos especialmente a los aspectos institucionales que puedan incidir en el logro de los fines, metas y objetivos de la misma" (Junta Universitaria, 1977).

Estas primeras políticas se dividieron en campos específicos que en cierta medida reflejan la situación actual heredada. Estas cinco primeras políticas fueron las siguientes:

- Política 1: Investigación de la clientela, pensada para el análisis de las características de los futuros usuarios de la UNED.

- Política 2: Investigación de necesidades y expectativas educativas, para el análisis de las áreas prioritarias que para la UNED "sea necesario desarrollar" en término de acciones para la formación y adiestramiento de recursos humanos.

- Política 3: Investigación de procesos de aprendizaje, para el análisis del proceso de enseñanza-aprendizaje en educación a distancia.

- Política 4: Evaluación del proceso de enseñanza-aprendizaje, que permita medir la calidad de dicho proceso en educación a distancia.

- Política 5: Evolución de los procesos administrativos y apoyo logístico, para medir la calidad y eficiencia de los procesos administrativos y de apoyo logístico. (Junta Universitaria, 1977)

La Oficina de Programación Curricular tenía las funciones de estructurar "un mecanismo eficaz para el diseño curricular de alta calidad" (Junta Universitaria, 1977: 25), en educación a 
distancia, elaborar perfiles profesionales y ocupacionales, organizar los planes de estudio, diseñar mecanismos para la evaluación de planes y programas de estudio y evaluarlos, y coordinar la evaluación de los servicios de tutorías y tutores, entre otras (Junta Universitaria, 1979). Esta fue una oficina de larga duración, que se reubicó en 1989 dentro de la Vicerrectoría Académica, por la reestructuración de la Dirección de Planeamiento Académico.

La Oficina de Investigación Institucional se encargaba principalmente de investigar las características de los estudiantes, las necesidades y expectativas del sistema de educación a distancia; identificar las necesidades de formación y capacitación; investigar los resultados de los métodos educativos relacionados con el sistema de educación a distancia y ejecutar y coordinar la investigación institucional en la UNED, entre otras (Junta Universitaria, 1979). Esta oficina también sufrió algunas modificaciones hasta que fue absorbida por la Dirección de Investigación en 1990.

En este sentido, se puede decir que la investigación llamada institucional pensada para el planeamiento académico y estructural de las actividades de la UNED se estableció de forma temprana, mientras que la investigación general inició en 1982, con la creación del Centro de Programas de Investigación. Por lo pronto, como se mencionó anteriormente, no es sino a principios de 1980 que la Junta Universitaria se comienza a plantear el tema de la investigación "no institucional".

Posteriormente, se propuso una estructura sencilla, de fácil constitución y de bajo costo y se desechó, en ese momento, la constitución de una Vicerrectoría de Investigación (como la de la Universidad de Costa Rica), ya que parecía "algo precipitado". La conformación de una Vicerrectoría de Investigación se ve problemática en ese entonces por las siguientes razones:

- No existe consenso.

- Puede llegar a convertirse en una estructura burocrática demasiado grande.
- Tiene un alto presupuesto.

- Una Vicerrectoría supone toda una organización estructural, direcciones, jefaturas y muchas cosas que se quisieron evitar en el caso de la investigación (Junta Universitaria, 1980).

Se decide, entonces, a favor de una estructura alternativa y se forma el Centro de Programas de Investigación, al cual se le adjunta un Consejo Técnico (COTI). Al director del Centro se da el rango de vicerrector y se adscribe el Centro de Programas de Investigación a la Rectoría, como fue inicialmente sugerido por la comisión encargada de dar las primeras ideas relacionadas con la investigación no institucional. El rango de Vicerrectoría de Investigación se mantuvo por un tiempo como lo evidencian algunos documentos de información financiera, donde claramente se usa el nombre de vicerrectoría.

El rango de vicerrector también se usó por mucho tiempo aún cuando para 1983 el Centro de Programas de Investigación (CPI) funcionaba completamente. En la dedicatoria de La tercera revolución educativa costarricense, al referirse a Enrique Góngora, primer encargado del CPI, Celedonio Ramírez hace mención de su participación en la UNED como "vicerrector de Investigación” entre 1981 y 1986 (Ramírez, 2006).

Las tareas de investigación dependerán administrativamente de la Rectoría y estarán a cargo de un funcionario con rango de vicerrector. Se nombrará un Consejo Técnico integrado por cinco miembros con atribuciones según se indica en el informe presentado por la Comisión. Los miembros externos de este comité recibirán una remuneración por los servicios que presten a la institución.

\section{Segunda etapa: de 1981 a 1986}

El primer encargado del Consejo de Programas de Investigación fue Enrique Góngora, y el primer Consejo Técnico estuvo formado por Jorge Manuel Dengo Obregón, Juan Manuel Esquivel Alfaro, Luis Fournier Origgi, Jorge Enrique Güier Esquivel y Eduardo Lizano Fait. 
El primer documento de políticas de investigación no institucional fue entonces el informe del plan de acción producido por este grupo, llamado "La Investigación en la UNED. Programa de acción, 1982-1984", el cual contenía esencialmente dos grandes partes: los antecedentes y el plan de acción en sí mismo (UNED, 1982).

En relación con los requerimientos financieros, se pensó en formar un fondo para financiar la investigación correspondiente a un $3 \%$ del presupuesto para el personal clasificado como académico que aumentaría hasta llegar a un $10 \%$ en 1985, así establecido en el plan de desarrollo de la UNED propuesto para el periodo 1982-1984. El monto inicial para el periodo 1982 se calculó en aproximadamente 400000 colones.

A pesar de lo básico de la propuesta, se pensaba que en el futuro la UNED establecería una política en materia de investigación clara y con objetivos igualmente bien delimitados. También se tenía la idea de ir elaborando propuestas similares para los siguientes periodos como el de 1985-1986 y sucesivamente. Sin embargo el proceso iniciado en 1980 quedó truncado y en el periodo entre 1984 y 1987 el Centro de Programas de Investigación no logró consolidar la posición de la UNED ni impactar a la comunidad costarricense como se quería, a pesar de algunos logros concretos.

En principio, en ese periodo se habla de unos ocho proyectos de investigación aprobados y financiados con los exiguos fondos destinados a investigación, que según información oficial de 1985, no alcanzaba al uno por ciento del presupuesto universitario, cerca de 1700000 colones (CIEST, 1985). A pesar de eso, se llegaron a realizar tres simposios sobre los problemas de la investigación y del desarrollo de la UNED, y se organizó lo que se conoció por cuatro años consecutivos como el "Setiembre Científico", que cautivó el "interés en la comunidad académica y cultural del país" (Brenes, 1987), entre otros alcances. Sin embargo, y en suma a los pocos recursos asignados a la investigación en la UNED, se debe mencionar que el CPI estuvo casi todo el año 1986 sin director, debido a que Enrique Góngora se jubiló. La falta de presupuesto y la inestabilidad en la dirección de la estructura a cargo de la investigación marcaron una tendencia, ya que a través de los años, el rubro de investigación destaca por tener la menor asignación presupuestaria en la UNED y la tasa de crecimiento más baja.

Con la llegada a la Rectoría de Celedonio Ramírez, se comenzó a dar una serie de reestructuraciones en la UNED, que también cubrieron el campo de la investigación.

Ya en 1983 se había dado la inclusión de artículos precisos referidos a la investigación en el Estatuto Orgánico de la UNED.

Para esa fecha el Estatuto Orgánico contenía las siguientes directrices:

- Artículo 1, inciso ch): Contribuir a la investigación científica y tecnológica para el progreso cultural, económico y social del país.

- Artículo 3, inciso c): Realizar e impulsar programas de investigación en áreas fundamentales para el desarrollo del país.

- Artículo 16, inciso b) (Funciones del Consejo Universitario): Determinar las políticas de la Universidad, aprobar los programas docentes de investigación y de extensión.

- Artículo 34: La investigación debe regirse por un orden de prioridades que guarde relación con los problemas presentes en el plano de la atención nacional y en forma referente realizarse en estrecha coordinación con otras instituciones.

- Artículo 35: La Universidad promoverá investigaciones tendientes a perfeccionar sus procesos de enseñanza-aprendizaje. (UNED, 1983)

Después de 1986, se tomaron algunas medidas en aras de fortalecer la investigación y darle una estructura sólida.

\section{Tercera etapa: 1987-1996}

Es hasta 1988 que se da la primera propuesta de crear una Vicerrectoría de Investigación en la UNED, impulsada por el rector Celedonio Ramírez. Uno de los primeros pasos que se dio en esta dirección fue el nombramiento del encargado del 
Centro de Programas de Investigación (CPI), que se había mantenido acéfalo por más de un año. Este nombramiento recayó en Albán Brenes.

En primera instancia, el doctor Brenes había sido nombrado para "diseñar un 'plan' o 'modelo' integral" para el desarrollo de la investigación en la UNED, al menos durante el quinquenio 19861990. Durante la realización de ese estudio y al estar la dirección del CPI vacante, se le pidió a Brenes presidir el Centro y el Consejo Institucional de Investigación CONIIN (antiguo COTI), de los cuales se hizo cargo en 1987 y donde elaboró, en conjunto con otros compañeros asesores, el Plan Anual Operativo del CPI en ese momento. Posteriormente, Brenes se abocó a la tarea original de diseñar el plan para la organización de la investigación que le había asignado el Consejo Universitario, el cual presentó, finalmente, en diciembre de 1987 con la colaboración de profesionales del CIEST, bajo el título "El desarrollo de la investigación en la UNED”.

Este documento está dividido en siete partes. En primer lugar define el concepto de investigación que la UNED podría llevar a cabo no sin antes poner en duda la efectividad de los términos investigación institucional e investigación científica, y de poner en evidencia la ambigüedad epistemológica que había existido en esta Universidad hasta el momento. Aunque estos términos se usaron desde los inicios de la UNED y se incluían en el plan de desarrollo 1986-1990, el documento de Brenes intenta darles formalmente, a las tareas de investigación en la UNED, parámetros epistemológicos más precisos, tomando como punto de partida la literatura disponible y lo establecido en el documento respectivo de lineamientos de políticas institucionales. Al respecto, Brenes sugiere la siguiente definición:

Se conceptúa la investigación como la indagación metódica que la UNED promueve en distintos campos del saber. Dicha indagación debe estar sustentada en métodos basados en operaciones lógicas, tomando como punto de partida datos objetivos. Es una tarea que realizan profesionales debidamente entrenados que poseen la actitud rigurosa, racional y sistemática que caracteriza a los científicos. Su propósito final es generar conocimientos o procedimientos aplicables al desarrollo institucional, científico, tecnológico y cultural de la Universidad y del pais (1987: 10).

Se rompe así una tradición en la UNED en la que las prácticas de investigación se veían en blanco y negro, como "institucional" contra "científica (o no institucional)". Después de dar esa concepción, Brenes se ocupa de determinar qué tipos de investigación sería deseable desarrollar en la UNED e instaura otro de los binomios conceptuales que gobernó el quehacer de la investigación en la UNED y su financiamiento desde entonces: la investigación interna y la investigación externa. Esto lo hace al determinar dos criterios de clasificación: los criterios de alcances y los criterios de propósitos.

En la segunda sección del documento, Brenes procede a sugerir una serie de políticas de investigación. En este sentido, es visionario y las políticas sugeridas pretenden, entre otras cosas, en forma general dar al quehacer de la investigación una permanencia que no había tenido hasta el momento y una presencia importante ante la comunidad universitaria. Para esto, él promulga la independencia y libertad académicas, aspectos que toman una relevancia significativa ante otros elementos tales como el rigor o la cientificidad de las investigaciones de las propuestas anteriores. Siete son las políticas esbozadas por Brenes (1987):

- La investigación como actividad permanente con programas específicos tan importantes como los programas docentes.

- Un organismo encargado de la investigación con una jerarquía igual en lo posible a la de los responsables de programas docentes, de planificación o de la administración.

- Representación de la UNED ante sistemas nacionales o internaciones que se dediquen a la ciencia y la tecnología.

- Libertad académica. 
- Infraestructura administrativa necesaria para ofrecer las condiciones necesarias a los programas de investigación.

- Independencia intelectual.

- Seguimiento de un orden de prioridades.

Después de sugerir las políticas macro del quehacer de investigación, Brenes describe los objetivos y funciones que debería tener el organismo de la UNED que eventualmente tendría a su cargo ese quehacer. Además, en una cuarta sección, describe con detalle los requerimientos de recursos humanos y materiales para el desarrollo de la investigación. Finalmente, Brenes sugiere una lista de programas y proyectos de investigación que se podrían realizar en la UNED, tomando en cuenta la definición de investigación interna dada en el documento.

Por otro lado, como parte de la celebración de su décimo aniversario en 1988, la UNED llevó a cabo su I Congreso Universitario de Educación a Distancia, en la primera semana del mes de febrero. Este primer congreso planteó conclusiones y recomendaciones sobre el tema de investigación que marcaron las políticas y acciones del quehacer durante la siguiente década.

A continuación se describirán las principales recomendaciones y logros:

\section{La investigación como quehacer}

El I Congreso de Educación a Distancia (UNED, 1988) da los primeros pasos para definir la investigación, la extensión y la docencia como quehaceres fundamentales y enfatizar en la integración de estos tres quehaceres. En materia de investigación y como política general de investigación se consigna "en forma expresa [...] la relación necesaria y permanente entre la investigación, la docencia y la extensión" (UNED, 1988: 84).

\section{Investigación interna e investigación externa}

En la mayoría de los aspectos, las conclusiones y recomendaciones emanadas del Congreso en materia de investigación, se basan en las ideas del Dr. Albán Brenes, quien además participó como conferencista. Ejemplo de esto es que se toma la decisión de dividir la investigación en dos campos de acción: la investigación externa y la interna. Además, como políticas generales también se orientan las acciones relacionadas al campo de investigación externa al desarrollo de investigaciones científicas y tecnológicas, y se agrega la dimensión de la investigación educativa sobre todo (y en esto la UNED mantiene una línea desde su fundación, la investigación sobre educación a distancia), además de que se insiste en que la investigación que se haga en la UNED debe responder a las necesidades prioritarias del país.

\section{Organización y administración de la investigación}

En relación con la organización y administración de la investigación, se propone un organismo que coordine e integre los esfuerzos y recursos de las dependencias que hacen investigación, que genere proyectos y que capacite a los funcionarios. Mucho más relevancia tiene el hecho de que, como parte de la organización de la investigación, se instruye a la administración superior de la UNED para que brinde los recursos necesarios, con el fin de que se cumplan los objetivos y acciones que sobre la investigación se señalan en el plan de desarrollo, para el quinquenio 86-90. También se define una serie de incentivos para estimular la investigación en la UNED tales como la licencia sabática y otros tipos de licencias y facilidades para que los funcionarios realicen sus proyectos de graduación sobre temas de interés para la UNED. Entre las recomendaciones, en relación con la estructura administrativa de la investigación, se hacen dos propuestas: 1) crear una Vicerrectoría de Investigación y 2) crear una Dirección de Investigación dentro de la Vicerrectoría de Planificación.

Al final, se decide crear una Dirección de Investigación, y no una Vicerrectoría. Razones presupuestarias y de infraestructura pesaron para esta decisión. A raíz de las conclusiones y recomendaciones del Congreso, el Consejo Universitario, en sesión n. ${ }^{\circ} 792-89$, del 15 de febrero de 1989, acuerda establecer la Dirección de Investigación, utilizando para ello la definición de 
investigación contenida y propuesta por Brenes en el documento citado, al igual que su tipología de investigación, siguiendo los criterios de alcances y de propósitos. Se definen así dos tipos de investigación, la interna y la externa, y tres más, la investigación básica, la aplicada y la de desarrollo tecnológico. Entre las funciones y objetivos de la nueva Dirección de Investigación se incluyen el promover, administrar y evaluar la investigación de la UNED, identificar temas y realizar investigaciones en campos relevantes para el desarrollo del país, conseguir los recursos para financiar programas de investigación y servir como vínculo entre la UNED y otras universidades (Consejo Universitario, 1989).

Con el establecimiento de la Dirección de Investigación se hacen también algunas modificaciones organizacionales en otras oficinas. Por ejemplo, se suprime la Dirección de Planeamiento Académico; se trasladan a la Dirección de Investigación la Oficina de Investigación Institucional y el Centro de Investigaciones Estadísticas y se suprime el Consejo Integrado de Investigación. Un cambio adicional que estaba esbozado en el borrador del acuerdo decía literalmente que "se adiciona al nombre de la Vicerrectoría de Planificación el término 'investigación' para denominarla, a partir de esta fecha, como: Vicerrectoría de Investigación y Planeamiento"; sin embargo, finalmente, este inciso se eliminó.

Además, el mismo acuerdo de la sesión $\mathrm{n}$. $^{\circ}$ 792-89 forma una comisión ad hoc con el fin de evaluar y perfeccionar las funciones de la Dirección de Investigación y de los centros y oficinas que a partir de entonces formarían parte de ella. Como punto de partida para la comisión se les indicó utilizar de nuevo el documento elaborado por el doctor Brenes. Esta comisión contaría con cuarenta y cinco días para emitir su pronunciamiento.

Dicha comisión ad hoc, coordinada por Juan Humberto Cevo, primer director de Investigación, presentó en el tiempo estimado un primer borrador en el que grosso modo da una definición más extendida de lo que se entendería por investigación en la UNED, y cuya modificación primordial consiste en darle el estatus de quehacer inherente a la actividad universitaria (Dirección de Investigación, 1989). Además de eso, reitera las categorías de investigación introducidas por Albán y define las líneas de trabajo como aquellos grupos de situaciones que configuran una cierta unidad problemática. Plantea también una serie de líneas de investigación interna tales como el planeamiento académico, el paquete instructivo, la vida estudiantil, deserción, rendimiento académico, entre otros. Existe a la vez una sección donde se plantea un esquema de interrelación entre la investigación y las otras funciones académicas, docencia y extensión. En la última sección de los borradores presentados, se hace una lista de políticas de investigación basadas de nuevo en el documento de Brenes de 1987 y se plantean los objetivos, funciones y la coordinación interna de la Dirección de Investigación. El documento borrador se conoce en sesión 80989 del 17 de mayo de 1989, donde los miembros del Consejo hacen señalamientos que muestran su disconformidad con algunos aspectos, como su ambigüedad e incertidumbre en relación con su presentación y contenido. En ese momento se decide formar otra comisión, para que se reúna con la comisión ad hoc y así aclarar las dudas surgidas.

Después de diferentes análisis, un año después, en sesión 857-90 del 7 de marzo del 1990 se aprueba un documento final llamado "De la Naturaleza de la Investigación en la Universidad", el cual define la investigación científica como quehacer inherente a la actividad universitaria y se definen dos nuevas vertientes para la investigación en la UNED:

a) La investigación educativa e institucional: o aquella que se dirige fundamentalmente al análisis de todos los aspectos relativos al proceso de enseñanza-aprendizaje a distancia y a su administración para la toma de decisiones.

b) La investigación general: o aquella que aborda problemas más amplios de orden nacional o universal (Consejo Universitario, 1990). 
A diferencia de los primeros borradores, en el acuerdo final se toman en cuenta reformas estructurales más profundas y, organizativamente, se establece que la investigación en la UNED será llevada a cabo por las siguientes dependencias (Consejo Universitario, 1990):

- Una Dirección de Investigación asesorada por un Consejo de Investigación.

- Un Centro de Investigación para el Desarrollo de la Educación a Distancia (CIDED), dependiente de la Dirección de Investigación.

- Un Centro de Investigación General (CIG), integrado por las cuatro cátedras básicas y dependiente de la Dirección de Investigación.

Este formato permaneció activo hasta 1997, cuando se estableció una profunda reestructuración de la Vicerrectoría de Planificación. Sin embargo, si se ven los informes de labores de la Dirección de Investigación y de la Vicerrectoría se pueden apreciar logros importantes en materia de investigación, al igual que algunos problemas de funcionamiento.

\section{Cuarta etapa: de 1997 a 2002}

Para mediados de los años noventa, la UNED entró en un periodo profundo de reorganización justificada por el objetivo de "hacer más eficiente, y más ágil la universidad, aprovechando mejor sus recursos humanos y generar economías que permitan introducir innovaciones" (UNED, 1998).

Con ese propósito, la Comisión del Consejo Universitario sobre el Desarrollo Organizacional recomienda, en 1995, la creación de una Comisión para la Reorganización Universitaria (Sesión 1153-95, articulo II, inciso 1), cuya principal función sería la de "modernizar la administración de la UNED, introduciendo en todo su quehacer acciones de cambio que aseguren la optimización de su capacidad de respuesta a los nuevos desafíos del entorno" (Consejo Universitario, 1996).

La Comisión de Reorganización presentó su informe final en abril de 1996 y ya para 1997 la reorganización de la Vicerrectoría de Planificación “estaba aprobada" (UNED, 1998), y se encontraban en marcha la reorganización de la Vicerrectoría Académica y el análisis de la reorganización de la Vicerrectoría Ejecutiva (Consejo Universitario, 1996).

Dicha reorganización afectó de manera muy directa el quehacer de la investigación que se encontraba desde 1990 bajo la administración de la Vicerrectoría de Planificación y en la cual se habían definido una Dirección de Investigación asesorada por un Consejo y, bajo su dependencia, dos Centros de investigación (el Centro de Investigación para el Desarrollo de la Educación a Distancia-CIDED y el Centro de Investigación General-CIG). Con esta reorganización toda la estructura de la investigación cambió.

Por consiguiente, la misma comisión propone que las funciones del CIG se adscriban a la Vicerrectoría Académica y que se cuente, para ese efecto, con un ente coordinador de la investigación general (UNED, 1996: 43). Igualmente se le asignan las funciones del Consejo de Investigación (CI) al Consejo Académico. En lo relacionado con el CIDED, y dentro del apartado sobre la reorganización de la Vicerrectoría de Planificación, la Comisión sugiere en primera instancia la transformación de esta "en una Vicerrectoría de Desarrollo" (UNED, 1996: 63), donde la investigación institucional conformaría una de las grandes áreas de acción para el desarrollo.

Como consecuencia, para la administración de la investigación educativa o institucional (interna) se formó el Centro de Investigación y Evaluación Institucional (CIEI), adscrito a la Vicerrectoría de Planificación (según acuerdo en sesión n. ${ }^{\circ}$ 1260-97). Igualmente, se instauró el Centro de Planificación y Programación Institucional (CPPI), para la formulación y organización de proyectos de inversión, la planificación de las actividades económicas anuales, y para la coordinación de los planes, programas y proyectos en la UNED (entre otros objetivos y funciones) (UNED, 1996: 63).

Con el fin de darles seguimiento a los proyectos de investigación externa o general se creó, adscrito a la Vicerrectoría Académica, el Centro 
de Investigación Académica (CIAC), que se especializó en temas relacionados con educación a distancia, educación abierta, planeamiento curricular, diseño instruccional, paquete instructivo, técnicas de estudio, orientación vocacional, entre otros. La creación del CIAC fue ratificada por el Consejo Universitario en sesión n. ${ }^{\circ} 1321$, artículo XII del 3 de abril de 1998, y se le da la misión de dedicar "al menos el 60\% de sus investigaciones a atender las solicitudes de las Escuelas, Dirección de Extensión Universitaria y Dirección de Posgrados," de integrar "a su labor las investigaciones de posgrado, de tal modo que algunas puedan atender los requerimientos de las Escuelas y Dirección de Extensión Universitaria" y de atender "las solicitudes de investigación que le haga el Consejo Universitario, el Consejo de Rectoría y los Consejos de Vicerrectoría" (Consejo Universitario, 1998). De muy corta duración el CIAC, luego dio paso al CEMPA, en el 2002.

Al mismo tiempo, en esa misma sesión, en su artículo VI se confirma la Dirección del Sistema de Estudios de Posgrado "con el objeto de permitir el desarrollo especializado de este tipo de programas en la UNED" y para que esta sea "responsable del apoyo logístico y administrativo a las escuelas para que estas planifiquen, diseñen y ejecuten los programas de posgrado" (Consejo Universitario, 1998). A su vez, el artículo VII describe los objetivos y las funciones de la Dirección del Sistema de Estudios de Posgrado.

Con la reestructuración, es definitivo que de nuevo la UNED, en materia de investigación, se volcó hacia una de carácter institucional o interna, y hacia la investigación académica enfocada a procesos de la educación a distancia, relegando el resto de áreas de investigación que se pueden definir como generales.

El II Congreso Universitario tiene una particular importancia en el desarrollo posterior de la investigación desde la reforma de la Vicerrectoría de Planificación en 1997 y, si se quiere, se constituye en el tercer gran esfuerzo institucional por elevar la práctica de investigación. El II Congreso Universitario determina que la investigación debe ser "aplicada a la realidad nacional y a las necesidades de los sectores menos favorecidos en nuestro país" (UNED, 2000), haciendo de los actores sociales parte integral de los procesos de investigación y logra una mejor articulación entre los diferentes quehaceres de la UNED en especial entre la investigación y la extensión, teniendo estos dos quehaceres la misión de proyectar a la UNED hacia la sociedad costarricense. En ese sentido se retoma una preocupación histórica en la UNED, derivada de su Ley de Creación: que la investigación científica esté vinculada con los problemas y retos nacionales.

El alcance de definición del quehacer de investigación en la UNED derivado del II Congreso Universitario es así mucho más amplio en términos de la investigación en sí y de sus tipologías. Se pasa de una dualidad interna-externa o institucional-disciplinaria, a fortalecer la promoción de la investigación en todas sus formas (UNED, 2000). Aun cuando este paradigma dual no se rompe por completo, la UNED entra, en relación con la investigación, en una coyuntura interesante y propicia para una reorganización de la investigación hasta ese momento inconclusa. A diferencia de intentos anteriores, donde la investigación se visualizaba en aislamiento y realizada solamente desde su rango de acción de espacio y tiempo (desde el CPI o la Dirección de Investigación), en este caso, se hizo un gran esfuerzo conceptual para integrarla a las acciones de los otros quehaceres, desde diferentes puntos y escenarios.

La moción 024 del II Congreso Universitario se refiere específicamente a la investigación y pretende fomentarla como "actividad académica fundamental del quehacer universitario" y dicta siete pasos para lograr su desarrollo (UNED, 2000).

Estas siete medidas se pueden agrupar en tres grandes grupos:

- las referentes a los incentivos laborales,

- las referentes a cambios en la estructura administrativa de la investigación

- y las relacionados con el estatus propiamente dicho de la investigación en la UNED. 
Aunque la moción 024 pretende diseminar, a todo nivel, el quehacer de la investigación, principalmente en las escuelas, esta moción también replica los intentos anteriores que procuraban la organización del quehacer por medio de una estructura encargada para tal efecto: el Consejo Institucional de Investigación. Específicamente, este Consejo sigue la misma lógica aplicada a la creación del CPI en 1980 y a la creación de la Dirección de Investigación en 1989. Por un lado se opta por una estructura institucional débil y centralizada en una rama del organigrama (el CPI de la Rectoría, la Dirección de Investigación de la Vicerrectoría de Planificación, el CIEI de la Vicerrectoría de Planificación y el CIAC de la Vicerrectoría Académica), enfatizando más bien la separación y conflicto histórico entre la investigación y la docencia. El nuevo Consejo Institucional de Investigación, como veremos más adelante, se ubicó en la Vicerrectoría Académica.

Igualmente, se optó por un ente rector y administrador de recursos de investigación, generador de políticas y de prioridades de investigación tal y como lo hicieron las previas comisiones en el caso del CPI, de la Dirección de Investigación y de instancias como el CIEI, el CIAC y otros. Si se comparan los documentos de la primera Comisión de Investigación de 1980 con el documento elaborado a petición del Consejo Universitario por el Dr. Albán Brenes y el resultado final a raíz del I Congreso Universitario que origina la Dirección de Investigación de 1990, con las prerrogativas de la moción 024 del II Congreso Universitario y las posteriores funciones asignadas al CII, veremos concurrencias importantes que han perpetuado una misma línea de organización de investigación en la UNED durante años.

En el acuerdo 1560 surge una nueva definición de investigación derivada de los lineamientos de política institucionales 2001-2006, que la define como las actividades para "la generación, renovación, ampliación y aplicación del conocimiento" (UNED, 2000a), muy diferente a la que hasta ese momento se había manejado. También, este acuerdo introduce un nuevo elemento a la fórmula de la estructura de la investigación, como lo son las comisiones de investigación (COMI) que se pueden organizar al seno de las escuelas, las direcciones, los centros universitarios y el SEP. Estas comisiones constituyen un esfuerzo para la descentralización de la actividad de investigación, con el fin de involucrar a un mayor número de funcionarios. Cada comisión de investigación contaba con un representante ante el Consejo Institucional de Investigación.

Las COMI son a su vez la microestructura a lo interno de cada unidad académica para la administración y la promoción de la investigación en la UNED. Su labor era coordinada por el Consejo Institucional de Investigación, órgano adscrito a la Vicerrectoría Académica, y con la función de ser en su naturaleza, un órgano asesor y decisorio en materia de investigación específicamente académica, además de tener la tarea de establecer políticas de investigación, analizar y aprobar o no los proyectos de investigación de las unidades académicas, definir procedimientos de aprobación de proyectos, generar lineamientos relacionados con la investigación, procurar financiamiento para las investigaciones, entre otras (Consejo Universitario, 2002).

De manera que, con el acuerdo 1560 , se pretende dar un giro radical a los procesos de investigación en la UNED, pero perpetuando de una u otra forma la división tradicional entre investigación institucional (CIEI) e investigación general (CII), e incluyendo en la fórmula una unidad de apoyo e investigación específicamente sobre la problemática de los procesos de enseñanza y aprendizaje y diseño curricular (CEMPA). También, el Acuerdo 1560 procura una mejor coordinación entre todos estos actores y lleva el quehacer de investigación a un nivel más micro, en el que todos los funcionarios tengan acceso a este, por medio de las comisiones de investigación de las diferentes unidades académicas. El órgano encargado de coordinar este conglomerado es el CII, que está integrado por representantes de cada uno de estos órganos. Además se agrega el Programa de Autoevaluación con el fin de mantener el elemento de calidad en todos los programas. 


\section{Quinta etapa: después del acuerdo 1560}

Desde el 2002, cuando se aprueba el acuerdo 1560 , se han dado otros pasos hacia el fortalecimiento de la investigación en la UNED, principalmente desde el CII y la Vicerrectoría de Planificación.

Otro proyecto que elaboró el CII en marzo del 2005 fue la "Propuesta para Fortalecer la Investigación en la UNED”, en el que se pretende dar de nuevo a la Vicerrectoría de Planificación el rol de órgano superior de representación política de la investigación y el CII se concibe como la instancia de conducción político-estratégica de la investigación.

Esta nueva fórmula para la investigación se basa en un modelo de Investigación, Innovación y Desarrollo $(\mathrm{I}+\mathrm{I}+\mathrm{D})$, de alta difusión entre universidades en la actualidad y que pretende el fortalecimiento de esos elementos (un modelo que en su momento se planteó ante las autoridades de la UNED). El documento presentado por el CII incluye además una propuesta para el impulso de la investigación desde las unidades académicas que reformula las funciones y responsabilidades de las COMI, y un paquete de incentivos para la investigación. La propuesta, en concordancia con el Acuerdo 1560, también promueve el establecimiento de centros e institutos de investigación.

Por otro lado, para enero del 2006, la Vicerrectoría de Planificación propuso una "Estructura ad hoc para la gestión de la investigación y la extensión en la UNED”, con el fin de responder a las previsiones del proyecto para los Lineamientos de Política Institucional 2006-2010, donde inicialmente se proponía la creación de la Vicerrectoría de Investigación y Extensión, con el fin de fomentar la investigación integrada a las necesidades sociales del país por medio de la extensión.

Otro hecho importante ha sido el acuerdo del Consejo de Rectoría en sesión n. ${ }^{\circ}$ 1413-2005, artículo II, celebrada el 11 de octubre del 2005, que establece el desarrollo del Centro de Investigación, Transferencia de Tecnología y Educación para el Desarrollo, ubicado en la propiedad de La Casona del asentamiento campesino La Perla, en
La Fortuna de San Carlos (adscrito a la Escuela de Ciencias Exactas y Naturales). Este tiene como fin operar una subsede del Centro Universitario de San Carlos para los programas que sean pertinentes a las necesidades de la región y con un personal de investigadores que estaría adscrito al Centro.

Con este panorama, en el 2006 se llevó a cabo la convocatoria para realizar el III Congreso Universitario bajo la noción del "Modelo de la UNED en una visión integradora" y cuyos resultados fueron coyunturales en materia de investigación. Por primera vez en la historia de la UNED, una mayoría amplia de congresistas lograron aprobar mociones encaminadas a la creación de una Vicerrectoría de Investigación y de la instauración de un sistema de investigación que incluya líneas de investigación, programas, redes e institutos. Aunque ninguna de las dos decisiones es nueva en la UNED, sí es la primera vez que las autoridades y una mayoría democrática decide impulsar la investigación desde una estructura definitoria como una Vicerrectoría.

En segundo lugar, el III Congreso Universitario establece la creación de una Vicerrectoría de Investigación. Para la creación de la Vicerrectoría se designa de formó temporal la figura de Director(a) de Investigación, con rango de Vicerrector, para que lleve a cabo la promulgación de la propuesta. El Consejo Universitario en sesión 1847-2007, del 19 de enero del 2007, acuerda designar a Katya Calderón Herrera como directora de Investigación, con el propósito de elaborar la propuesta de la Vicerrectoría. Esta Dirección de Investigación comenzó a funcionar en febrero del 2007 y su tarea inicial fue la conformación del documento que justificara la creación de la Vicerrectoría.

Por último, el III Congreso Universitario es coyuntural porque propone la investigación como un sistema que debe articular "todos los esfuerzos individuales y colectivos con el fin de elevar el nivel y la calidad de la investigación" (UNED, 2006). Originalmente, la tarea de investigación se visualizó, primero, para el financiamiento y el 
patrocinio en forma de programas; para ahora finalmente, articularlo como un sistema.

El primer resultado tangible de las mociones del III Congreso Universitario en materia de investigación ha sido la transformación sufrida por el Centro para el Mejoramientos de los Procesos Académicos (CEMPA), el cual se suprime a partir del acuerdo 1846-2006 del 15 de diciembre del 2006. En su lugar, este acuerdo aprueba la creación de dos instancias: la primera es el Programa de Apoyo Curricular y Evaluación de los Aprendizajes (adscrito a la Vicerrectoría Académica) "con la finalidad de que brinde el asesoramiento en materia curricular y evaluación de los aprendizajes que requieren los programas de pregrado, grado y posgrado de la Universidad"; la segunda instancia es el Programa de Investigación en Fundamentos de la Educación a Distancia, que temporalmente queda adscrito a la Rectoría, "mientras se crea la Vicerrectoría de Investigación, solicitada por la Moción 06 del III Congreso Universitario" (UNED, 2006).

\section{Conclusiones}

El desarrollo de la investigación en la UNED-Costa Rica ha estado íntimamente relacionado con la evolución de su administración y de los esfuerzos efectuados por la institución para la promoción, la organización y la inversión de recursos en investigación. Como hemos podido observar, dicha evolución ha estado marcada además por una serie de situaciones, documentos y tipos de organización cuya genealogía es rastreable al analizar de cerca el conjunto de evidencias documentales legadas en los archivos, bibliotecas y acuerdos institucionales a todo nivel. Por esta genealogía es que nos hemos atrevido a caracterizar la historia crítica de la administración de la investigación en la UNED en cinco momentos específicos.

La creación de la Vicerrectoría de Investigación es un paso trascendental para el establecimiento de relaciones de todos los procesos de la UNED, ya que permite que esta institución pueda establecer con mucho mejores criterios las áreas de desarrollo, no solo en materia de investigación, sino también de docencia y extensión. También le concede a la UNED una mejor representación ante organismos interuniversitarios y de financiamiento. Le permite de manera más eficiente la gestión, la promoción y ejecución de programas, proyectos e iniciativas de investigación que nazcan del seno universitario; le permite evaluar de una mejor manera todos los procesos de investigación, elevando así la calidad de las investigaciones y su pertinencia en el desarrollo del país en general y del desarrollo de las comunidades en específico, además de cumplir efectivamente con los mandatos incluidos en su ley de creación, su estatuto orgánico y en su misión y visión.

\section{Bibliografía}

Brenes, A. (1987). La investigación en la UNED. San José.

Centro de Investigación y Estadística-Ciest. (1985). Anuario estadístico 1984. San José.

Consejo Universitario de la Uned. (1989). Sesión n. $792-$ 89. San José.

Consejo Universitario de la Uned. (1990). "De la naturaleza de la investigación en la UNED”. En Sesión n. ${ }^{\circ} 857$ 90, 7 de marzo. San José.

Consejo Universitario de la Uned. (1996). Sesión n. ${ }^{\circ} 1153$ 95, artículo II, inciso 1, 14 de julio. San José.

Consejo Universitario de la Uned. (1997). Sesión n. ํ 126097, 19 de marzo. San José, Costa Rica.

Consejo Universitario de la Uned. (1998). Sesión n. ${ }^{\circ} 1321$ 98, artículos VI y XII, 3 de abril. San José.

Consejo Universitario de la Uned. (2002). Sesión n. ${ }^{\circ}$ 15602002, 15 de marzo. San José.

Consejo Universitario de la Uned. (2005). Sesión n. ${ }^{\circ} 1413$ 2005, artículo II, 11 de octubre. San José.

Consejo Universitario de la Uned. (2007). Sesión n. ${ }^{\circ} 1847$ 2007, 26 de enero. San José.

Dirección de InVESTigación. (1989). Circular 89-013, 31 de marzo. San José.

Junta Universitaria. (1977). "Políticas de investigación institucional de la UNED”. Sesión n. ${ }^{\circ} 35,27$ de setiembre. San José: CIDREB.

Junta Universitaria. (1979). Plan de Desarrollo de la UNED 1979-1983. Documento n. ${ }^{\circ}$ 507. San José: EUNED. 
Junta Universitaria. (1980). Sesión n.ํ224-80. San José: CIDREB.

RAmírez, C. (2006). La tercera revolución educativa costarricense: Memoria de la creación y puesta en marcha de la UNED (1977-1982). San José: EUNED.

Universidad Estatal a Distancia. (1976). Ley de Creación de la UNED. San José.

Universidad Estatal a Distancia. (1982). La investigación en la UNED: Programa de acción, 1982-1984. San José: Oficina de Publicaciones de la UNED.

Universidad Estatal a Distancia. (1983). Estatuto Orgánico. San José: Oficina de Publicaciones de la UNED.
Universidad Estatal a Distancia. (1988). I Congreso Universitario de Educación a Distancia. San José.

Universidad Estatal a Distancia. (1996). Informe Comisión de Reorganización Institucional, abril. San José.

Universidad Estatal a Distancia. (1998). Informe de labores 1986-1997. San José: EUNED.

Universidad Estatal a Distancia. (2000). Mociones aprobadas. Sesión plenaria. II Congreso Universitario. San José. [Documento institucional]

Universidad Estatal a Distancia. (2000a). Lineamientos de política institucional 2001-2006. Asamblea Universitaria. San José. 
